\title{
A Protocol for the Determination of the Growth Rate of Organisms Subject to Interrupted Exponential Growth
}

\section{Upton $\mathrm{G}^{*}$}

Department of Mathematical Sciences, University of Essex.

${ }^{*}$ Corresponding author: Upton G, Department of Mathematical Sciences, University of Essex, E-mail: gupton@essex.ac.uk

Citation: Upton G (2014) A Protocol for the Determination of the Growth Rate of Organisms Subject to Interrupted Exponential Growth. J Comp Sys Bio 1(1): 103. doi: 10.15744/2455-7625.1.103

Received Date: October 17, 2014 Accepted Date: October 30, 2014 Published Date: December 03, 2014

\section{Introduction}

A standard laboratory procedure with cultures that grow exponentially involves regular dilution (so that the organism is never nutrient limited). When the logarithm of growth is plotted against time, exponential growth then appears as a series of parallel lines. An investigation of the dependence of the growth rate on the ambient conditions may involve switching the culture from one set of conditions to another. In such a situation the culture may require an acclimation period before beginning growth at its revised exponential rate: the resulting plot will start uncertainly, but end with the required straight line. However, if the change in conditions is too great, or the new conditions are close to the limiting conditions that the organism can tolerate, then the culture may suffer complete or partial death: in these cases there may be no exponential growth
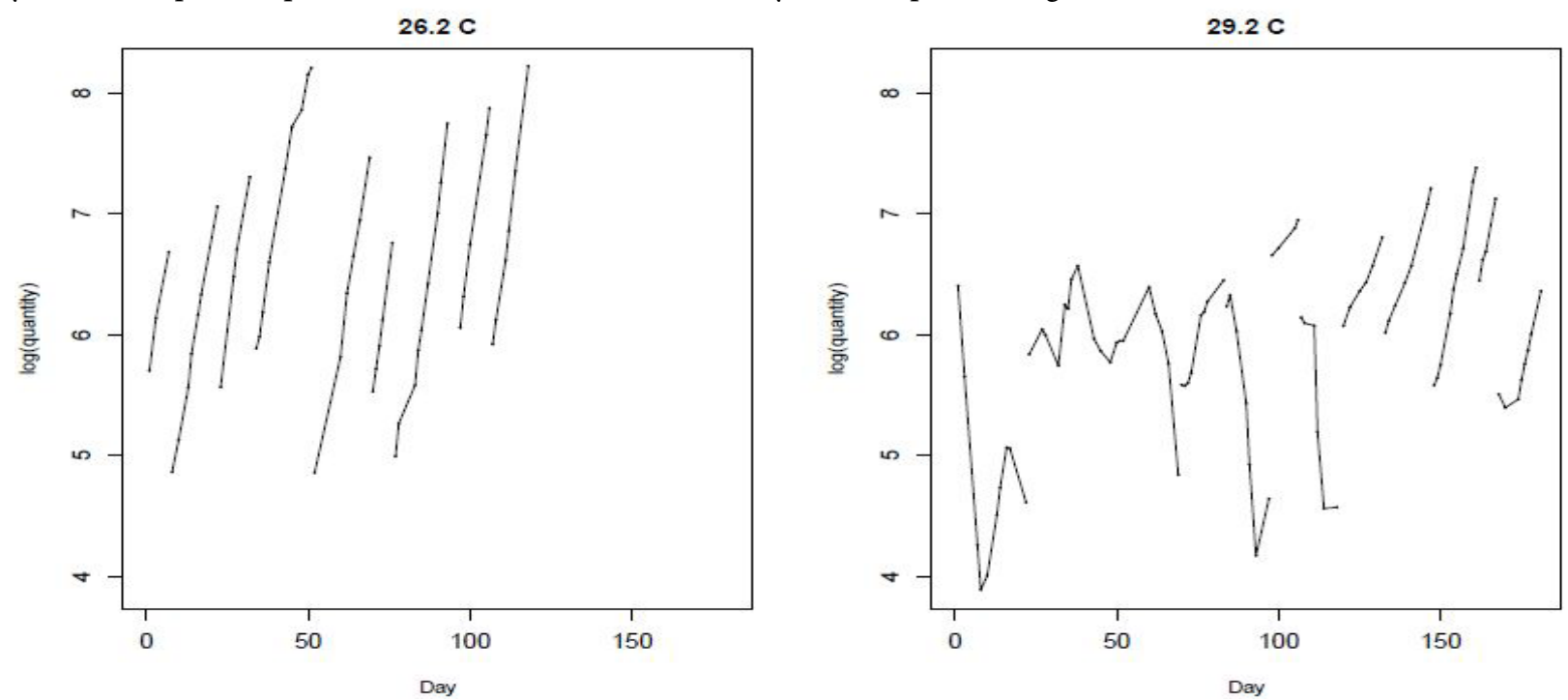

Figure1: Two examples of the growth of laboratory cultures of the cyanobacteria Trichodesmium erythraeum IMS101. In both cases the concentration of $\mathrm{CO}_{2}$ is $180 \mathrm{ppm}$ and the light intensity is $400 \mu \mathrm{mol} \mathrm{m}^{-2} \mathrm{~s}^{-1}$. The examples differ in respect of the constant ambient temperatures $\left(26.2{ }^{\circ} \mathrm{C}\right.$ and $\left.29.2^{\circ} \mathrm{C}\right)$ under which the organism is grown

Figure 1 illustrates data obtained in the laboratory relating to the growth of the cyanobacteria Trichodesmium erythraeum IMS101. Under favourable conditions $\left(26.2^{\circ} \mathrm{C}\right)$ Trichodesmium grows strongly and the plot shows the ideal pattern of near-parallel lines. Any procedure for combining the information from these nine separate growth segments would arrive at apprroximately the same estimate of the growth rate, with a very low associated standard error. However, under the same conditions but with an ambient temperature of $29.2^{\circ} \mathrm{C}$, growth is less assured: of the eleven segments, five include falls. There are seven segments that end in continuing increases in growth, but these noticeably vary in slope. It is not obvious which segments of the plot should be used, nor how the information from those segments should be combined. It is the purpose of this short paper to propose a protocol for the estimation of the growth rate from such a set of heterogeneous segments. Using such a protocol frees the analyst from accusations of bias.

\section{The protocol}

1. A segment is potentially informative if it terminates with at least three increasing observations. In the cases illustrated only four segments are not potentially informative. 
2. Within a potentially informative segment the selected data consists of the largest sequence of consecutive observations (including the final observation) that is reasonably collinear. The definition of 'reasonably collinear' is arbitrary: we used the requirement that the value of the multiple correlation coefficient, $\mathrm{R}^{2}$, for the fit of a linear model through the logged observations, should be greater than $\mathrm{R}_{\text {crit }}^{2}$. We took $\mathrm{R}_{\text {crit }}^{2}=99 \%$.

3. A segment with a selected sequence that has a slope that differs significantly from the estimated common slope of the sequences from the remaining segments is discarded. The definition of 'significantly' is arbitrary: we used a $1 \%$ significance level. This requirement is applied iteratively. If there are several segments with slopes that differ significantly from the common slope of the remaining segments, then it is the sequence associated with the most significant difference that is discarded. After the discard all the remaining sequences are re-tested, and this continues until either there are no sequences with discordant slopes, or until there are just two sequences left. In the latter event, if the sequences have significantly differing slopes, then no value can be returned.

\section{Example}

To illustrate the protocol we use the results for the $29.2{ }^{\circ} \mathrm{C}$ data illustrated as part of Figure 1 . Of the 11 segments, four were terminated because the amount of the culture was not increasing. The remaining seven segments were terminated by dilution. The data for these seven are given in Table 1. All seven end with at least three increasingly large observations,and are therefore potentially informative. However, the first two of these segments fail to meet the collinearity criterion since the maximum values of $\mathrm{R}^{2}$ for these segments are $97.9 \%$ and $98.9 \%$. The selected $\left(\mathrm{R}^{2}>99 \%\right)$ sections of the remaining segments are shown in bold in Table 1 . The summary of the selected data in Table 2 includes the slopes of the best fit lines. These slopes range between 0.075 and 0.143 .

\begin{tabular}{|c|c|c|c|c|c|c|c|c|c|c|c|c|}
\hline Day & 70 & 71 & 72 & 73 & 76 & 77 & 78 & 83 & - & 98 & 100 & 105 \\
$\log ($ quantity) & 5.59 & 5.58 & 5.60 & 5.69 & 6.16 & 6.19 & 6.28 & 6.45 & - & 6.66 & 6.72 & 6.88 \\
\hline Day & 106 & - & 120 & 122 & 125 & 127 & 129 & 132 & - & 133 & 134 & 136 \\
$\log ($ quantity) & 6.95 & - & 6.08 & 6.23 & 6.36 & $\mathbf{6 . 4 3}$ & $\mathbf{6 . 5 7}$ & $\mathbf{6 . 8 1}$ & - & 6.02 & 6.12 & 6.24 \\
\hline Day & 139 & 141 & 146 & 147 & - & 148 & 149 & 150 & 153 & 154 & 155 & 157 \\
Log(quantity) & $\mathbf{6 . 4 3}$ & $\mathbf{6 . 5 7}$ & $\mathbf{7 . 0 9}$ & $\mathbf{7 . 2 1}$ & - & $\mathbf{5 . 5 8}$ & $\mathbf{5 . 6 5}$ & $\mathbf{5 . 7 5}$ & $\mathbf{6 . 1 8}$ & $\mathbf{6 . 3 8}$ & $\mathbf{6 . 5 0}$ & $\mathbf{6 . 7 2}$ \\
\hline Day & 160 & 161 & - & 162 & 163 & 164 & 167 & - & 168 & 170 & 174 & 175 \\
Log(quantity) & $\mathbf{7 . 2 7}$ & $\mathbf{7 . 3 8}$ & - & $\mathbf{6 . 4 5}$ & $\mathbf{6 . 6 2}$ & $\mathbf{6 . 6 9}$ & $\mathbf{7 . 1 3}$ & - & 5.51 & 5.40 & $\mathbf{5 . 4 7}$ & $\mathbf{5 . 6 3}$ \\
\hline Day & 176 & 177 & 178 & 181 & - & & & & & & & \\
Log(quantity) & $\mathbf{5 . 7 6}$ & $\mathbf{5 . 8 7}$ & $\mathbf{6 . 0 1}$ & $\mathbf{6 . 3 6}$ & - & & & & & & & \\
\hline
\end{tabular}

Table 1: The data for the seven potentially informative segments at $29.2^{\circ} \mathrm{C}$. The selected data (having $\mathrm{R}^{2}>99 \%$ :) are shown in bold. Each segment is terminated by a - sign.

The final step of the protocol consists of discarding segments having slopes that differ significantly from the common slope of the parallel lines fitted to the remaining potentially informative segments. The penultimate column of Table 2 reports the tail probabilities associated with these tests applied to the five remaining segments. The critical value specified by the protocol was $1 \%$. Two segments show markedly different slopes from the remainder, with the outstandingly different segment being that from days 148 to 161. It stands out because it has the steepest slope of the five and, crucially, contains the most observations over the longest period of time: its slope is therefore not only extreme, but also strongly confirmed as such. By comparison, the segment with the lowest rate of increase has just three observations spread over just 5 days.

Removal of a segment from consideration, especially when that segment had many observations, makes a considerable difference to the tail probabilities at the next iteration. Since it was a steep segment that was removed, the two least steep segments now differ less from the common slope of the remainder. Indeed, all four tail probabilities now exceed 1\%, implying that a common slope can be fitted without further discard. The estimate of the common slope is 0.109 , with a standard error of 0.006 .

\begin{tabular}{|c|c|c|c|c|c|c|}
\hline \multirow{2}{*}{ Segment (Days) } & \multirow{2}{*}{$\begin{array}{l}\text { Number of } \\
\text { observations }\end{array}$} & \multirow{2}{*}{$\begin{array}{l}\text { Number } \\
\text { selected }\end{array}$} & \multicolumn{2}{|c|}{ Selected observations } & \multicolumn{2}{|c|}{ Tail probabilities (see text) } \\
\hline & & & Length (days) & Estimated slope & First iteration & Second iteration \\
\hline $70-83$ & 8 & 0 & & & & \\
\hline $98-106$ & 4 & 0 & & & & \\
\hline $120-132$ & 6 & 3 & 5 & 0.075 & 0.0105 & 0.0212 \\
\hline $139-147$ & 4 & 4 & 8 & 0.099 & 0.0023 & 0.1260 \\
\hline $148-161$ & 9 & 9 & 13 & 0.143 & 0.0001 & - \\
\hline $162-167$ & 4 & 4 & 5 & 0.133 & 0.9579 & 0.1196 \\
\hline $168-181$ & 8 & 6 & 7 & 0.126 & 0.8268 & 0.0333 \\
\hline
\end{tabular}

Table 2: Results for the selected data in the seven potentially informative segments at $29.2^{\circ} \mathrm{C}$ 


\section{Acknowledgement}

I am very grateful to Toby Boatman and Richard Geider of the Department of Biological Sciences at the University of Essex for bringing this problem to my attention.

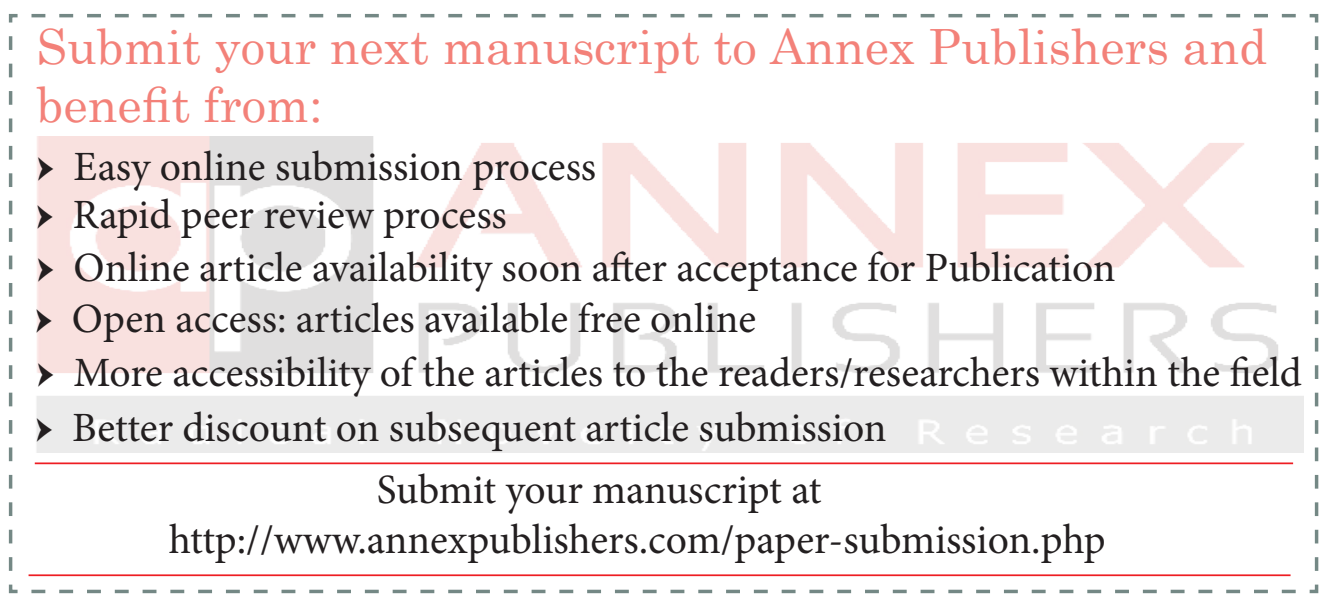

